\title{
Environmental Injuries: Hyperthermia and Hypothermia
}

\section{Amanda McDonald, Rebekah Stubbs, Prince Lartey, \& Shaeleigh Kokot}

Environmental injuries have the potential to be life threatening. The most common activitymediated environmental injuries reported are hyperthermia and hypothermia (Noe et al., 2012). Although hyperthermia and hypothermia can be fatal, both conditions are preventable. These types of injuries occur year-round through a variety of activities, affect multiple systems within the body, and result from a combination of internal and external factors. Noel et al. (2012) reported that within one year, there were 19,000 hospital visits attributed to heat and cold exposure. Due to the frequent occurrence of these injuries, it is important to understand the mechanisms of how and why they occur, the effect they have on the body, and what indicates whether an athlete is suffering from heat or cold exposure. Medical personnel on site should know how to reduce the risk of injury and implement immediate treatment protocols (Cappaert et al., 2008).

In the treatment of hyperthermia, it is important to know how it develops in the body, as well as how the body reacts to it. An athlete can become hyperthermic when metabolic heat production exceeds heat dissipation. One of the ways the body reacts is thermoregulation, which is a contributing aspect of homeostasis, and a temperature regulation system which involves both heat production and heat loss (Kjertakov \& Epstein, 2013).

The normal body temperature of the average individual is near $37^{\circ} \mathrm{C}$, depending on other factors such as the time of day and body composition (Kjertakov \& Epstein, 2013). However, as the core temperature of an individual rises past a normal range, there are potentially dangerous implications if the condition is left untreated, including death. Exertional Heat Stroke (EHS) is one of the leading causes of death among athletes, yet it is "100\% preventable" (Pryor, 2013, p. 268).

Athletes are particularly susceptible to heat related illnesses due to the combination of intense activity that occurs in hot and humid environments. Football and long-distance running are common activities where athletes may experience hyperthermia due to sport-specific intrinsic and extrinsic factors. Intense exercise can increase the metabolic rate of the athlete by 15-20x (Kjertakov \& Epstein, 2013). These factors can result in heat mediated illness when the body is unable to "sustain the rate of cardiac output necessary to simultaneously meet the demands of blood flow for exercising muscle, skin, and vital organs" (Kjertakov \& Epstein, 2013, p. 135).

Dehydration in hot conditions can increase peripheral vasoconstriction, comprising and impairing the functions of the brain, skeletal muscles, and systemic blood flow (Trangmar \& González-Alonso, 2019). This may also result in less cerebrovascular flow, decreasing "the level of central cognitive or neuromuscular drive", weakening the descending neural pathways or inhibiting motor neurons (Cheung \& Sleivert, 2004, p. 106). Therefore, hyperthermia affects the athlete's cardiovascular and nervous system when the body's demand for oxygen cannot be

Vol. 4(1) | DOI: https://doi.org/10.31542/muse.v4i1.1854 


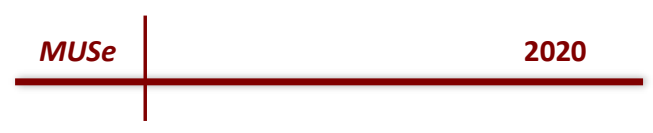

met. A decrease in oxygen and nutrient supply to body organs such as the brain, heart, and skeletal muscles could explain accelerated fatigue and impairment (González-Alonso \& Trangmar, 2019). Additionally, the body is only about $25 \%$ efficient, meaning that as much as $75-80 \%$ of the energy produced by the body is released as heat. In a hyperthermic individual, heat remains trapped in the body, dramatically increasing core temperature (Desforges \& Simon, 1993).

The human body has many coping mechanisms used to cool the body and dissipate excess body heat. These mechanisms include both behavioural and autonomic regulatory systems. Behavioral temperature regulation is when a conscious adjustment to regulate the body temperature is made, in response to deviations away from the body's shell (outer layer) temperature, which is identified through peripheral thermosensors (Flouris, 2009). Examples of a behavioural regulation response to an increased outer temperature may include the decision to sit down and rest, move into the shade, or to wear certain clothing (Periard et al., 2015). Rising core temperatures stimulate central thermoreceptors, activating the autonomic thermoregulatory process. Core temperature is regulated by the autonomic nervous system, meaning that humans do not consciously have to regulate their body temperature (Cheung \& Flouris, 2009). The thermosensors of the body provide afferent input to thermoregulatory control centers located in the preoptic nucleus of the anterior hypothalamus (Cheung \& Flouris, 2009). With heat exposure, the thermal center generates a signal though efferent fibers in the autonomic nervous system. This signal results in cutaneous vasodilation and increases the rate of sweat production, redistributing the blood from the core to the skin (Periard et al., 2015). Behavioural regulation is usually the first cooling mechanism generated since it is activated prior to changes in the body's core temperature. The autonomic regulatory system starts when the behavioural response fails to maintain a stable core temperature (Flouris, 2009).

Hyperthermia progresses in a gradual process that can be hierarchically categorized in stages based on severity: heat cramps, heat exhaustion, and heat stroke. Heat cramps are an early indication of dehydration, which is caused by water loss and an imbalance of electrolytes such as salt, calcium, and potassium. It is common for the athlete to complain of cramping in the muscles that are specifically recruited during activity. These cramps can worsen into heat exhaustion if left untreated, due to dehydration and a prolonged high core temperature (Kjertakov \& Epstein, 2013). This high core temperature "occurs when the thermoregulatory system becomes overwhelmed due to excessive heat production (ie, metabolic heat production from the working muscles) or inhibited heat loss" (Casa et al., 2015, p. 987). Heat exhaustion is identified by the presence of excessive sweating, cool and clammy skin, and a rapid pulse. The athlete may also experience some light-headedness and a decrease in cognition (Kjertakov \& Epstein, 2013). If untreated, heat exhaustion may evolve to heat stroke, requiring immediate medical attention. Major symptoms include a core temperature equal to $40.5^{\circ} \mathrm{C}$ or higher, confusion, unconsciousness, or shock. The athlete may also present with nausea, loss of coordination, and hot, sweaty skin. This contrasts with the classic, more passive type of heat stroke that "typically affects children and the elderly, who present with dry skin" (Casa et al., 2015 , p. 990). The main way to distinguish between these different stages of hyperthermia is to assess core body temperature using a rectal thermometer (Kjertakov \& Epstein, 2013).

Although exertional heat illness is not always preventable, many intrinsic and extrinsic risk factors can be modified to ensure the athlete's safety (Pryor, 2013). Intrinsic predisposing 


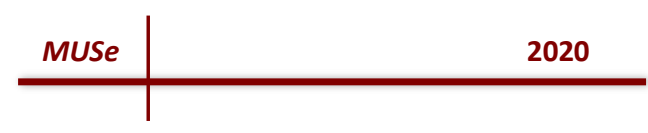

factors of hyperthermia include one's weight, lack of heat acclimatization, hypohydration, dehydration, skin disorders, other congenital disorders, infection, and supplements (Pryor, 2013). Individuals with poor fitness levels, commonly seen in overweight and obese football linemen, typically work harder to keep up with their fit teammates and end up generating more internal heat (Pryor, 2013). Since fat acts as an insulator, it inhibits heat from escaping the body, making these individuals more susceptible to heat stroke. Cheung \& Sleivert (2004), suggested that athletes with more aerobic training and lower levels of adipose tissue have an increased ability to tolerate a higher core temperature. Therefore, athletes at the start of their season, when they are usually deconditioned, typically reach a point of heat exhaustion earlier than they would later in their season, when their fitness level would have increased.

Gradually introducing an individual or athlete to heat exposure over a 10-14 day period at the start of the season allows the body to make physiological adaptations (Pryor, 2013). Heat acclimatization allows one's body to decrease their internal body temperature and heart rate, while increasing stroke volume, sweat output, and sweat evaporation (Pryor, 2013). Since many sports are played outdoors, it is especially important to allow the body to adapt to any hot or humid conditions present. The National Collegiate Athletic Association adopted heat acclimatization guidelines for college football in 2003 to prevent EHS (Pryor, 2013). It is important to have a set of guidelines in place for athletes to reduce their risk of becoming hyperthermic. This is especially important for American football players because "they are the most vulnerable athletes for exertional heat illnesses" (Grundstein et al., 2017, p. 1471). For example, heat acclimatization guidelines for preseason football states that for the first five practices you can only have one practice a day for 3 hours. Of those practices, one or two should be non-contact, and the amount of equipment worn should be reduced to only wearing a helmet. The second week of practices can include 2 practices every other day, athletes can wear full equipment, and play with full contact (Pryor, 2013).

When conditions are favourable for hyperthermia (high heat and humidity index), it is important to recognize the symptoms of heatstroke and to be able to effectively treat the athlete. Coaches and athletic trainers should provide "access to water for all athletes to minimize fluid losses based on individual fluid needs" (Adams, 2019, p. 6). To ensure proper hydration, the athlete should consume $500-600 \mathrm{~mL}$ of water or a sports drink 2-3 hours before exercise, as well as 200-300 mL 10 to 20 minutes prior to activity (Casa et al., 2000). During post exercise hydration, it is important to replenish any fluid loss within 2 hours of completion of the exercise. Electrolytes can be added to the athlete's water to speed up the process of hydration (Casa et al., 2000). Without appropriate replenishment of fluids before and during exercise, hypohydration and dehydration can increase the risk for EHS (Pryor, 2013). Dehydration of 1\% to $2 \%$ of body weight begins to compromise physiological functions and negatively influence performance. Dehydration greater than $3 \%$ of body weight further disrupts physiological functions and increases one's risk of developing exertional heat illnesses (Casa et al., 2000).

When dehydrated, cardiac stroke volume decreases, which can place stress on the thermoregulatory system to maintain heat balance (Pryor, 2013). Athletes in a hot external environment can sweat 1-2 $L$ an hour, and most athletes drink less than they sweat (Desforges \& Simon, 1993). Athletes suffering from dehydration tend to have a higher internal body temperature because of impaired blood flow and altered sweating responses. Surface blood 


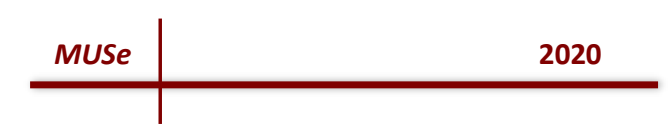

vessels constrict to conserve blood volume which decreases sweat output (Casa et al., 2000).

There are various other intrinsic factors that predispose one to hyperthermia such as skin disorders, infections, drugs, and dietary supplements. Ectodermal dysplasia and anhidrosis are skin disorders that impair the ability to sweat, which limits thermotolerance (Pryor, 2013). Infection may also reduce one's thermotolerance by increasing systemic activation of cytokines (Pryor, 2013). Although drugs and dietary supplements may further impair sweating and negatively impact thermotolerance, consumption of these substances have the potential to affect various other body mechanisms, increasing the risk of EHS. These additional mechanisms include impaired cardiovascular performance, disturbances in water and electrolyte balance, increased heat production, and decreased perception of fatigue (Pryor, 2013). Examples of these drugs and dietary supplements include anticholinergic and antiepileptic agents, antihistamines, decongestants, phenothiazines, tricyclic antidepressants, amphetamines, ergogenic stimulants, lithium, diuretics, $\beta$-Blockers, and ethanol (Pryor, 2013).

In addition to intrinsic risk factors, there are also extrinsic factors such as: extreme heat and humidity (which are measured by WBGT (wet-bulb globe temperature)), low work to rest ratios, excess equipment or clothing, and poor medical supervision (Pryor, 2013). WBGT is the gold-standard for assessing environmental heat stress placed on individuals during physical activity. WBGT measures the ambient temperature, relative humidity, wind, and solar radiation to monitor environmental conditions (Cooper et al., 2017). Athletic trainers can monitor WBGT levels to delay sporting events, and schedule rest and water breaks when levels are above 28 (Pryor, 2013). The intensity of the exercise coupled with high temperatures should dictate the "frequency and duration of rest breaks" (Pryor, 2013, p. 270). During breaks, the individual should be encouraged to take off excess equipment, such as their helmet and padding, and rest in the shade while consuming an adequate amount of fluid (Pryor, 2013).

As the temperature rises, the environmental temperature becomes hotter than the skin, which strains the body as heat dissipation relies heavily on evaporation (Pryor, 2013). Equipment and clothing can act as a barrier to evaporation by "creating a microenvironment between the skin and the uniform" (Pryor, 2013, p. 270). Clothing has the potential to become soaked with sweat, as is often the case for overfat athletes who tend to generate more heat. Saturated cloth interferes with the evaporation of sweat, comprising the body's main mechanism of heat loss. In addition, excessive equipment and dark clothing should be limited when exercising in hot environments, as dark colors absorb more heat (Pryor, 2013).

The case study of the late Korey Stringer, a defensive lineman for the Minnesota Vikings, is a particularly powerful example demonstrating how these internal and external risk factors interact. On August 1, 2001, he "became the first and to date the only professional American football player to die from exertional heat stroke" (Grundstein et al., 2017, p. 1471). Stringer was involved in a full day training session, and in the afternoon, he began to experience nausea and vomited. A physician on site diagnosed Stringer as experiencing heat exhaustion. The next day, Stringer participated once more in intense training sessions, despite being strongly advised to not continue training (Grundstein et al., 2017).

There were several factors that promoted the development of heat stroke in this player. The environmental conditions were particularly hot and humid, and Stringer wore full gear, restricting the release of body heat. At the time, Stringer weighed 335 pounds, 45 pounds over 


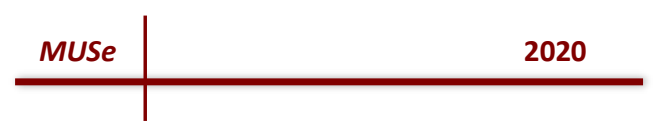

his normal playing weight. This resulted in the production of more heat, and the overfatness inhibited the dissipation of heat. Stringer continued to train despite showing signs of distress. After the training session had been completed, he collapsed and was rushed to the hospital before any cooling attempts were made. Upon arrival at the hospital his core temperature was $42.67^{\circ} \mathrm{C}$ (Grundstein et al., 2017). Later information was released that Stringer was using supplements which contained ephedra, increasing his risk of developing thermo-regulatory disorders and of experiencing "strokes, seizures, and heart arrhythmia" (McCallum \& Munson, 2002, p. 56).

Stringer's death raised awareness of heat illness in the athletic community, and it put emphasis on the importance of recognition and treatment. Both the National Athletic Trainers Association and American College of Sports Medicine recommend using rectal temperature as an accurate and reliable reading of core temperature (Pryor, 2013). However, there are less invasive methods that seem to pertain to sport such as "aural, tympanic, oral, temporal, forehead, and axillary" (Pryor, 2013, p. 271). Any reading above $40.5^{\circ} \mathrm{C}$ is EHS and is considered a medical emergency (Adams, 2019). Immediate medical attention is required because the longer the core temperature is above $40.5^{\circ} \mathrm{C}$, the greater the chance of death or long-term implications (Pryor, 2013). Athletic trainers should always be present at games and practices as they can monitor and treat symptoms of hyperthermia. Although most athletic trainers have read the Casa et al. (2015) position statement on exertional heat illnesses, only $18.6 \%$ have used rectal thermometers to assess core body temperature, and less than half have used cold water immersion to treat EHS (Mazerolle, 2010).

If the rectal temperature is taken and is below $40.5^{\circ} \mathrm{C}$, it is recommended that an individual is immediately removed from play. One might not always have their core temperature taken, but it is important to monitor athletes and be aware of the signs and symptoms of exertional heat exhaustion. If these symptoms appear, the athlete is expected to take off any excess equipment or clothing, consume liquids, elevate feet and rest in the shade until they cool down (Pryor, 2013). Removing an individual at play can prevent exertional heat exhaustion from turning into a medical emergency.

Cold water immersion $(\mathrm{CWI})$ is reported to produce the fastest cooling rates and is commonly used to treat athletes with EHS (Pryor, 2013). CWI involves placing an individual into a cold-water ice bath, which typically has a water temperature of about $10.0^{\circ} \mathrm{C}$ (Casa, 2010). Ice packs should be placed on any limbs that are not submerged in the water (Pryor, 2013). During $\mathrm{CWI}$ immersion treatment, rectal temperature should be monitored to ensure the athlete is cooling sufficiently (Pryor, 2013). The gold standard for cooling time is to have the individual cool down by $0.22^{\circ} \mathrm{C} \cdot \mathrm{min}-1$ (Adams, 2019). It is critical that the patient is cooled in less than thirty minutes to prevent any life-threatening implications (Pryor, 2013). Once the rectal temperature reaches a temperature of $38.5^{\circ} \mathrm{C}$, it is safe to remove the patient from the $\mathrm{CWI}$ immersion treatment.

Alternatively, other cooling modalities have been shown to be effective in treating individuals with EHS. Tarp assisted cooling (TACo) is an abbreviated version of cold water immersion which reduces body temperature above the accepted cooling rate (Adams, 2019). In the absence of a stationary cooling tub, TACo can serve as an alternative method to treat EHS on-site. TACo utilizes a tarp and allows the individual to lay in a semi-recumbent position while ice and water is poured over their body (Adams, 2019). Further cooling modalities used to treat 


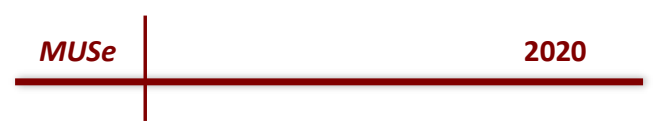

exertional heat exhaustion include placing ice packs on main arteries, misting, or seated rest (Pryor, 2013). However, these methods do not provide optimal cooling in 30 minutes and can therefore not be used to treat individuals with EHS (Pryor, 2013). Ironically, excessive cooling may result in hypothermia.

Hypothermia is an environmental injury that is common for athletes participating in cold environments such as skiing, ice-skating, biathlon, and numerous other sports. Hypothermia occurs when the body's internal temperature drops to $35^{\circ} \mathrm{C}$ or below. It is often caused when the athlete is exposed to rain, wind, water, or snow for a prolonged amount of time, and the body loses more heat than it can produce. To reduce the number of people affected each year, it is important to raise awareness and inform people on the symptoms and the mechanisms involved in the process. Hypothermia "affects all physiological systems in the body" (Fernandez \& Valdez, 2012, p. iii). Severe cases of hypothermia can lead to irreversible bodily changes such as a loss of motor function and possibly even death. All three stages of hypothermia (mild, moderate, and severe) can cause damage to the body and will be discussed in terms of severity and the changes occurring in the body during the stages (Fernandez \& Valdez, 2012).

Hypoxia, the deterioration of tissue due to a lack of blood flow, is one of the major concerns of hypothermia. The lack of blood flow is caused by vasoconstriction mediated by the cold. The body loses about $90 \%$ of its heat through the skin (Fernandez \& Valdez, 2012). To attenuate this, blood vessels constrict and send less blood to the periphery. The body's autonomic system puts preference on keeping the internal organs and brain supplied with blood and oxygen, which in turn means areas such as the hands and feet receive less blood and are susceptible to frostbite. In a process called shivering, muscles begin to contract uncontrollably, creating heat to rewarm the body. Shivering is the body's final mechanism to maintain heat, and when hypothermia progresses past the mild stage, shivering stops and the body starts to shut down to try to preserve its vital organs (Fernandez \& Valdez, 2012).

The neurological damage that can stem from hypothermia can result in total loss of movement. This was the case for skier Anna Bågenholm, a woman who lost control while skiing and ended up with her head underwater for over an hour. Her body reached an internal temperature of $13.7^{\circ} \mathrm{C}$, the lowest recorded temperature of a survivor (Jeican, 2014). She suffered cardiac arrest and was rushed to a hospital, where she was revived with the help of a heart lung-machine that pumped out her blood and rewarmed it. Bågenholm suffered severe nerve damage and lost her ability to move for close to a year. Her case was rare, but significant, and demonstrates the impact that severe hypothermia may have on a person's life (Froze to death and lived, 2018).

The brain is one of the most sensitive areas in relation to hypothermia. Decreased blood flow to the brain can cause ischemia and cerebral hypoxia, with possible symptoms being drowsiness, numbness, and loss of balance. Slower metabolism coupled with the lower levels of oxygen in the brain are key reasons why people demonstrate a lower level of responsiveness and may experience hallucinations. The decreased amount of oxygen in the brain randomly activates neurons in the visual cortex in the occiput, resulting in impaired vision and reports of seeing bright lights (Jeican, 2014). There are many recorded cases of people who were discovered nude in the cold due to paradoxical undressing, a potential side effect of hypothermia. It is believed that after the initial vasodilation occurs, if the body continues to lose heat, vasodilation occurs and the sudden increase in blood to the body makes the person feel 


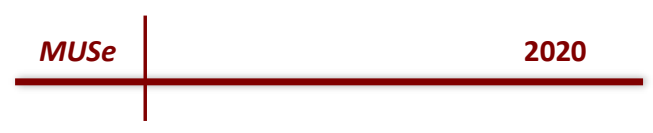

warm (Desclous et al., 2017). This, along with the impaired mental state, is likely the reason for the bizarre phenomena that has been linked to more severe cases.

The body's first response to the cold is to oppose it, as seen by an increase in metabolism in the early stages of cold related injuries. Increased metabolism, as well as the increase in cardiac output, both work to increase body heat in an attempt to reach the body's natural internal temperature of $37^{\circ} \mathrm{C}$. As the severity of hypothermia increases, the heart develops tachycardia as well as an increase in cardiac output and blood pressure. The latter stages (moderate and severe) show a decrease in heart rate up to 50\% (Fernandez \& Valdez, 2012). Hypothermia also depresses ATPase, reducing the amount of active calcium pump units, and causing possible delays in the heartbeat (Fernandez \& Valdez, 2012). As the hypothermia progresses, it is not uncommon for patients to develop a ventricular arrhythmia, and in severe cases, cardiac arrest (Balik et al., 2019). The respiratory system is another target of hypothermia. The respiratory system follows the same order of steps as metabolism; when mild hypothermia occurs around the $35^{\circ} \mathrm{C}$ mark, a person's breathing will increase. When a person's internal body temperature drops to around $30^{\circ} \mathrm{C}$, hypothermia depresses respiratory activity and decreases the amount of breaths a person will take per minute (Fernandez \& Valdez, 2012).

Due to the invasive and detrimental effects hypothermia has on the body, the best treatment plan for this cold injury is to take preventative measures and lower the risk of hypothermia altogether (Fudge, 2016). Before any engagement in sport, environmental conditions should be monitored, and measures should be taken specific to the type of activity being performed. For example, the International Ski Federation cancels events if the temperature drops below $-20^{\circ} \mathrm{C}$, and triathlons are cancelled if water temperature decreases past $11.7^{\circ} \mathrm{C}$. Other than cancelling competitions, triathlon events are also modified to include shorter swim durations if water temperatures are between $11.7^{\circ} \mathrm{C}$ and $13.3^{\circ} \mathrm{C}$ (Fudge, 2016). The Wind Chill Temperature Index (WTC) estimates the ambient temperature that would have equal heat loss rates compared to conditions with convective wind currents (Fudge, 2016). Therefore, the WTC is used to predict the potential risk of sustaining a cold injury, because it recognizes that it is important to monitor the effect of exposed skin and cold wind in outdoor environments (Cappaert et al., 2008).

Additional environmental factors to be considered include the increased risk during darkness, water immersion, and at higher altitudes (Cheung et al., 2016). The absence of sunlight eliminates a main type of radiant heat energy that the body otherwise absorbs. Water submersion conducts heat faster than air and therefore increases heat loss. Activity in low water temperatures requires extra energy, as more oxygen uptake is needed to thermoregulate. The air is colder and holds less water at high altitudes, meaning that the body undergoes more insensible water and respiratory heat loss to saturate and warm inspiratory air to body temperature. Hypoxic conditions at high altitudes also correlate to increased metabolic energy demands during performance, competing with the energy required for thermoregulation (McArdle et al., 2016).

Interactions with different environmental conditions impact which mechanism of body heat loss is heightened. The human body loses heat via conduction, convection, evaporation, and radiation (Cappaert et al., 2008). Conductive heat loss occurs in any type of moist environment, including snow or rain (Cappaert et al., 2008). Arguably the most significant risk is water immersion, as conductive heat loss is $25 x$ greater in water than in air (Macaluso et al., 


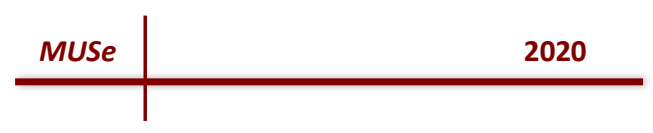

2011). Even if an athlete is not immersed in water, but continues to be in contact with wet clothing, their heat loss can increase by $5 x$ (Cappaert et al., 2008). Convection heat loss is particularly important to consider during activities that produce man-made wind, such as biking, skiing, and running. Convection breaks down the thermal gradient, and the insulation benefits from wearing proper clothing becomes relatively ineffective (Fudge, 2016). Removing this thermal barrier also enhances evaporative heat loss because the amount of exposed skin is increased (Cappaert et al., 2008). Radiation heat loss is also more active with uncovered skin. However, in contrast to other mechanisms, wind or water does not affect radiation heat loss (Cappaert et al., 2008). When all forms of heat loss are combined, body heat transfer can be as great as 70x higher in water compared to dry conditions (Cappaert et al., 2008).

To reduce the risk of hypothermia, athletes should be prepared going into competitions (Fudge, 2016). One method of preparation includes following the recommendation to dress in 3 proper layers of clothing. The internal layer should be moisture-wicking and designed to allow for evaporation of sweat (Cappaert et al., 2008). The goal of the internal layer is to maintain an insulating air layer adjacent to the skin (Fudge, 2016). It has been suggested that this internal layer be made of polyester, polypropylene, or even synthetic wool material (Cappaert et al., 2008; Fudge, 2016). These materials are effective because they possess sweat-wicking abilities, so moisture does not interfere with the insulating air layer. Wool is also able to retain heat, even when wet (Fudge, 2016).

A middle clothing layer, typically made from wool or fleece, should be worn to provide insulation (Cappaert et al., 2008). Finally, the athlete should come prepared with a removable external layer that is wind and water resistant (Cappaert et al., 2008). When deciding on an external layer, the athletes should be aware that the more water-proof material, the less breathable the layer may be, and it can potentially trap moisture (Fudge, 2016). Also, it is recommended that the external layer is only worn in wet or windy conditions, and that it is exchanged with dry clothing when possible. Otherwise, the athlete may overheat, and a wet outer layer can comprise the functionality of the other two layers (Fudge, 2016).

Along with these recommendations, the athlete should avoid heavy, bulky clothing as it can reduce mobility and increase metabolic costs (Cheung et al., 2016). This puts the athlete at a higher risk of becoming hypothermic, as well as limits optimal performance. In general, as the intensity of the activity increases, rectal and axillary temperatures increase, therefore less clothing is necessary (Macaluso et al., 2011). However, under wet or windy conditions, exercise alone does not provide enough protection for digits of the body (Fudge, 2016).

Other areas that are important to cover include toes, fingers, ears, and the head (Cappaert et al., 2008). To help keep distal extremities warm and reduce the effects of peripheral vasoconstriction, cover the core and other larger areas of heat exchange (Fudge, 2016). Wearing an extra pair of socks can sometimes help keep the athletes toes warm. However, the athletes should consider wearing larger shoes, so the fit is not too tight. This tightness should be avoided because it can constrict blood flow to the feet and decrease foot perfusion (Fudge, 2016). Fudge issues a note of caution when using chemical hand warmers, as they have been shown to increase the risk of cold injury. An athlete may perceive that they are warm when they may have inadequate protection (Fudge, 2016).

Although hypothermia can be developed by any person, various intrinsic factors such as sex and age make individuals more susceptible to injury. Despite women typically having more 


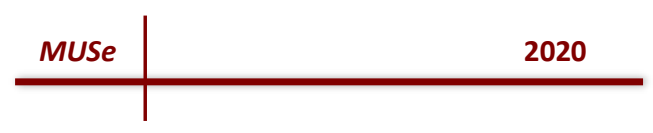

subcutaneous fat to help with insulation, if two people of the opposite sex have equivalent subcutaneous fat levels, women tend to have a larger surface area to mass ratio. This increases the rate of heat loss and makes women twice as vulnerable to sustaining a cold injury compared to men (Cappaert et al., 2008). An increased rate of heat loss is also what explains the heightened risk of cold injury for individuals of both young and old age. Prepubescent children lose more heat because they tend to have more surface area compared to body mass (Cappaert et al., 2008). Adults over 50 are at a greater risk because the sympathetic nervous system response to vasoconstrict is diminished with age, allowing more blood to travel to the skin and dissipate heat (Cappaert et al., 2008). In addition, older individuals are commonly less active, resulting in an increase of body fat and blood pressure, as well as a decrease in muscle mass. This raises one's body mass index and lowers metabolic heat production, directly correlating with a declined shivering response for thermoregulation (Fudge, 2016).

Pre-participation medical exams are also important to determine the extent to which an individual may be at risk of a cold injury (Cappaert et al., 2008). In terms of the activity itself, pre-screening tests can be done to understand the fitness factors involved. Based on a performance standard acceptable for that particular activity, the physical demands and workload of a task can be calculated (Cheung et al., 2016).

Conditions that increase the risk of sustaining a cold injury include Raynaud syndrome, anorexia nervosa, and cardiovascular disease (Cappaert et al., 2008). In Raynaud syndrome, blood vessels spasm, decreasing blood flow to the extremities (Cappaert et al., 2008). Anorexia nervosa is also associated with peripheral vasoconstriction, as well as insufficient body fat and a decreased metabolic rate (Cappaert et al., 2008). Cardiovascular disease increases the risk of becoming hypothermic because the combination of cold and exercise emphasizes the demand placed on the cardiovascular system. This additional demand is characterized by increased blood pressure and myocardial oxygen needs. Cardiovascular disease may also be associated with decreased cutaneous and subcutaneous blood flow (Cappaert et al., 2008).

Additional conditions have been found to interfere with total body heat production and thermoregulation. For example, the shivering response of thermoregulation can be impaired if the athlete is hypoglycemic (Fudge, 2016). Chronic skin conditions, hyperhidrosis, and burns can also increase the risk of injury due to heightened body heat loss (Fudge, 2016). Medications affecting the nervous or vascular system can also interfere with the athlete's ability to thermoregulate. For example, benzodiazepines can cause a delay in the body's response time to thermoregulate, and beta blockers that increase vasoconstriction also increase the risk of cold injury (Fudge, 2016).

Prior to competitions, athletes may also attempt cold acclimatization to decrease the risk of becoming hypothermic (Cheung et al., 2016). The rationale for cold acclimatization is that it will effectively help decrease the body's vasoconstriction response to ensure adequate circulation and combat tissue damage (Cappaert et al., 2008). In order to undergo hypothermic habituation, the athletes should spend longer durations of time in moderately cold temperatures, rather than expose themselves to extreme cold temperatures for short periods. This procedure should be carried out over consecutive days for at least 2 weeks (Cappaert et al., 2008). Although cold acclimatization has been shown to be beneficial, it is highly individualized, considered more difficult to accomplish compared to heat acclimatization, and should not be the sole method used as a preventative measure (Cappaert et al., 2008). 


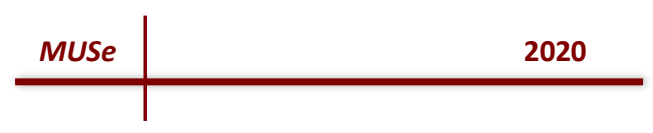

Preparatory steps that are suggested for any kind of activity include educating trained personnel on site, coaches, and athletes about proper risk reduction, recognition, and treatment protocols for hypothermia (Cappaert et al., 2008). Protocols should be designed specifically for practice and game situations as well as adjusted based on environmental conditions before and during activity (Cappaert et al., 2008). Rewarming supplies and sheltered facilities should be readily available. Examples of supplies needed on site include warm ingestible fluids, heat packs, blankets, external heaters, extra dry clothing, a rectal thermometer, a telephone, and a warm tub or whirlpool for warm water immersion treatment (Cappaert et al., 2008).

If preventative measures fail and an athlete presents signs and symptoms of hypothermia, immediate action must be taken. Wet clothing should be removed immediately and replaced with dry warm clothing or blankets (Cappaert et al., 2008). Remove the athlete from windy and wet environments to a sheltered facility (Cappaert et al., 2008). The main methods for treating mild hypothermia is to use rewarming supplies like warm blankets and warm fluids (Jeican, 2014). Food should also be provided, preferably with $6-8 \%$ carbohydrate composition, for the individual to maintain metabolic heat production (Cappaert et al., 2008). During rewarming, heat should mainly be applied to the core and large areas of heat exchange like the armpits and groin. If heat is applied to the extremities, the athlete may be more at risk of rewarming afterdrop. Afterdrop occurs when blood vessels in the extremities dilate, causing cold blood to be sent to the core, decreasing core body temperature (Cappaert et al., 2008). In extreme cases this phenomenon may lead to cardiac arrhythmia or even death (Cappaert et al., 2008). Hypothermia suppresses the ATPase enzyme which in turn hinders calcium pump activity necessary for myocardial conduction. A resulting low intracellular concentration of calcium impairs cardiac contractibility and ultimately decreases the body's cardiac output (Jeican, 2014).

Methods to treat mild hypothermia should also be applied to help a patient with moderate or severe hypothermia. Athletes should be moved gently as rough handling has been associated with spontaneous cardiac arrhythmias (Fudge, 2016). If an athlete is in cardiac arrest, the priority is to regain normal sinus rhythm and perform CPR (Fudge, 2016). If the environment is less than ideal and proper supplies are not available, consider postponing the rewarming process until in a safe environment (Fudge, 2016). Implement active external rewarming techniques such as warm water immersion, electric blankets, and external heaters (Jeican, 2014). More aggressive techniques can also be used by a physician in a hospital setting. These techniques may include heated intravenous fluids, cardiopulmonary bypass, and hemodialysis (Jeican, 2014). Physicians must be prepared for any post rewarming complications, such as renal failure or infection (Cappaert et al., 2008).

When hyperthermia or hypothermia treatment is complete, rehabilitation protocols should be implemented to clear the athlete to return to play. Each protocol is highly individualized as the injury is related to the amount of time spent above or below the critical level (Pryor, 2013). The longer the individual's core temperature remains above or below the critical level, the longer the recovery time will be (Pryor, 2013). Before returning to significant physical activity, the individual should be "asymptomatic and all blood tests have returned to a reference range" (Pryor, 2013, p. 274). In general, to be cleared to play the athlete should show similar strength, aerobic fitness levels, range of motion, proprioception, and psychological conditions as they displayed before the injury. 
An understanding of the physiological and behavioural changes in the body upon hyperthermia or hypothermia is crucial when preparing how to reduce the risk of these injuries, as well as when implementing a treatment program. Not only knowing how the body changes, but knowing why these changes occur is important so that the athlete can take proper preparation measures prior to competing. Increased risk of these injuries should be monitored, and activity should be adjusted accordingly based on environmental conditions and predisposing individual characteristics. If an athlete sustains either of these environmental injuries, treatment should aim to return the athlete to a normal core body temperature of around $37^{\circ} \mathrm{C}$. Once the athlete is stable, and normal physical and cognitive functioning is restored, the athlete may be cleared to continue their activity provided that they are cautious and watch for signs and symptoms of possible recurrence. 


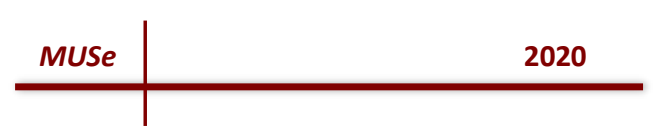

\section{References}

Adams, W., Belval, L., Berg., A., Hosokawa, Y., Stearns, R., Casa, D. (2019). Exertional heat stroke of Max Gilpin: A preventable death. Quest, 72(1), 102-115.

https://doi.org/10.1080/00336297.2019.1637355

Balik, M., Porizka, M., Matousek, V., Brestovansky, P., Svobodova, E., Flaksa, M., ... Belohlavek, J. (2019). Management of accidental hypothermia: An established extracorporeal membrane oxygenation centre experience. Perfusion, 34(1S), 74-81. https://doi.org/10.1177/0267659119830551

Cappaert, T., Stone, J., Castellani, J., Krause, B., Smith, D., \& Stephens, B. (2008). National Athletic Trainers' Association position statement: Environmental cold injuries. Journal of Athletic Training, 43(6), 640-658.

Casa, D., Kenny, G., Taylor, N. (2010). Immersion treatment for exertional hyperthermia: Cold or temperate water? Medicine \& Science in Sports \& Exercise, 42(7), 1246-1252. https://doi.org/10.1249/MSS.0b013e3181e26cbb

Casa, D. J., Demartini, J. K., Bergeron, M. F., Csillan, D., Eichner, E. R., Lopez, R. M., ... Yeargin, S. W. (2015). National Athletic Trainers' Association position statement: Exertional heat illnesses. Journal of Athletic Training, 50(9), 986-1000. https://doi.org/10.4085/1062-6050-50.9.07

Casa, D., Armstrong, L., Hillan, S., Montain, S., Reiff, R., Rich, B., ... Stone, J. (2000). National Athletic Trainers' Association Position Statement: Fluid replacement for athletes. Journal of Athletic Training, 35(2), 212-224. Retrieved from http://nata.org

Cheung, S., Flouris, A. (2009). Reviewing the functional architecture of the human thermoregulatory system. Body Temperature Regulation, 25-64. Canada: Department of Physical Education and Kinesiology, Brock University.

Cheung, S., Lee, J., \& Oksa, J. (2016). Thermal stress, human performance, and physical employment standards. Applied Physiology, Nutrition, and Metabolism, 41(6), 148-164. https://doi.org/10.1139/apnm-2015-0518

Cheung, S., Sleivert, G. (2004). Multiple triggers for hyperthermic fatigue and exhaustion. Exercise and Sport Sciences Reviews, 32(3), 100-106. https://doi.org/10.1097/00003677200407000-00005

Cooper, E., Grundstein, A., Rosen, A., Miles, J., Ko, J., Curry, P. (2017). An evaluation of portable wet bulb globe temperature monitor accuracy. Journal of Athletic Training, 52(12), 1161-1167. https://doi.org/10.4085/1062-6050-52.12.18

Descloux, E., Ducrot, K., Scarpelli, M., Lobrinus, A., \& Palmiere, C. (2017). Paradoxical undressing associated with subarachnoid hemorrhage in a non-hypothermia case? 


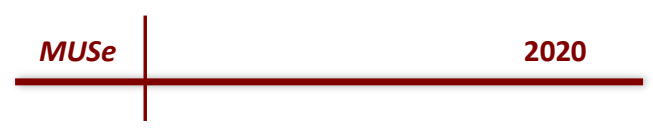

International Journal of Legal Medicine, 131(5), 1341-1345.

https://doi.org/10.1007/s00414-017-1597-3

Desforges, J., Simon, H. (1993). Hyperthermia. The New England Journal of Medicine, 483487.

Fernandez Garza, V. G., \& Valdez Delgado, J. I. (2012). Hypothermia: Prevention, recognition and treatment. New York: Nova Science Publishers, Inc. Retrieved from

http://search.ebscohost.com.ezproxy.macewan.ca/login.aspx?direct=true\&db=e000xna\& $\underline{A} \mathrm{~N}=541031 \&$ site $=$ ehost-live \&scope $=$ site

Flouris, A. (2009). Fundamental principles governing behavioural thermoregulation. Body Temperature Regulation, 1-24. Trikala, Greece: Institute of Human Performance and Rehabilitation.

Froze to death and lived - Anna Bågenholm. (2018, November 23). SOS Hydration. Retrieved from https://soshydration.com.au/anna-bagenholm-froze-to-death-but-lived/.

Fudge, J. (2016). Exercise in the cold: Preventing and managing hypothermia and frostbite injury. Sports Health, 8(2), 133-139. https://doi.org/10.1177/1941738116630542

Grundstein, A., Knox, J., Vanos, J., Cooper, E., \& Casa, D. (2017). American football and fatal exertional heat stroke: A case study of Korey Stringer. Int J Biometeorol, 61(1), 14711480. https://doi.org/10.1007/s00484-017-1324-2

Hyperthermia. (2019). Retrieved from https://sportmedbc.com/article/hyperthermia.

Jeican I. I. (2014). The pathophysiological mechanisms of the onset of death through accidental hypothermia and the presentation of "The Little Match Girl" case. Medicine and Pharmacy Reports, 87(1), 54-60. https://doi.org/10.15386/cjm.2014.8872.871.iij1

Kjertakov, M., Epstein, Y. (2013). Exertional heat stroke in athletes. Open Access Macedonian Journal of Medical Sciences, 1(1), 135-139. https://doi.org/10.3889/oamims.2013.026

Macaluso, F., Felice, V., Boscaino, G., Bonsignore, G., Stampone, T., Farina, F., \& Morici, G. (2011). Effects of three different water temperatures on dehydration in competitive swimmers. Science \& Sports, 26, 265-271. https://doi.org/10.1016/j.scispo.2010.10.004

Mazerolle, S., Scruggs, I., Casa, D., Burton, L., McDermott, B., Armstrong, L., Maresh, C. (2010). Current knowledge, attitudes, and practices of certified athletic trainers regarding recognition and treatment of exertional heat stroke. Journal of Athletic Training, 45(2), 170-180. Retrieved from https://library.macewan.ca/full-record/s3h/48811711

McArdle, W.D., Katch, F.I., and Katch, V.L. (2016). Essentials of exercise physiology. (5 ${ }^{\text {th }}$ ed.), Philadelphia, PA: Wolters Kluwer.

McCallum, J., \& Munson, L. (2002). A question of life and death. Sports Illustrated, 97(4), 56. Retrieved from https://library.macewan.ca/full-record/a9h/7029804 


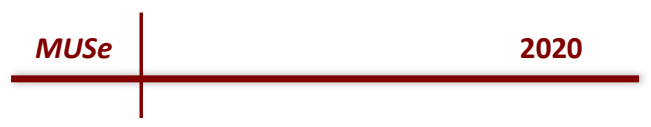

Periard, J., Racinais, S., \& Sawka, M. (2015). Adaptations and mechanisms of human heat acclimation: Applications for competitive athletes and sports. Scandinavian Journal of Medicine \& Science in Sports, 25(S1), 20-38. https://doi.org/10.1111/sms.12408

Noe, R. S., Jin, J. O., \& Wolkin, A. F. (2012). Exposure to natural cold and heat: Hypothermia and hyperthermia Medicare claims, United States, 2004-2005. American Journal of Public Health, 102(4), e11-e18. https://doi.org/10.2105/AJPH.2011.300557

Pryor, R., Casa, D., Holschen, J., O'Connor, F., Vandermark, L. (2103). Exertional heat stroke: Strategies for prevention and treatment from the sports field to the emergency department. Clinical Pediatric Emergency Medicine, 14(4), 267-278. https://doi.org/10.1016/j.cpem.2013.10.005

Trangmar, S., González-Alonso, J. (2019). Heat, hydration and the human brain, heart and skeletal muscles. Sports Medicine, 49(1), 69-85. https://doi.org/10.1007/s40279-0181033-y 